\title{
Analysis of commercial drone sounds and its identification
}

\author{
Sinwoo Yoo \\ Kwangwoon University \\ Republic of Korea \\ sinwooyoo@kw.ac.kr
}

\author{
Hyukjun $\mathrm{Oh}$ \\ Kwangwoon University \\ 01897 \\ Republic of Korea \\ hj_oh@kw.ac.kr
}

\begin{abstract}
The usage of quadcopter types of drones is now on mature and a practical stage and many major manufacturers are expanding its applications into various regions with it. Considerable characteristic of this type of flying object as its maneuverability and practicality is now being focused on how we control this among our urban life from the possibility of any offensive usage. Most of them are either small enough to avoid many current airborne detection methods and cheap enough to use them as disposable. In this paper, we tried to analyze the recorded sounds of a subset of commercial quadcopter types of drones and built a trained simple non-linear neural network filter to classify them among the given sound samples. We borrowed Mel-frequency cepstral coefficients as the well-known methodology of sound analysis process but including some of the parameter adjustments for this research, and applied LeNet neural network filter structure for the following classification test. To maintain the information of adjacent samples among the series of wave samples, 2-D spectrogram planning was applied as for the input signal preprocessing. Most of the frequencies from drones were observed as gathered around 3 to $5 \mathrm{Khz}$, up to around $10 \mathrm{Khz}$, and adjusted LeNet architecture could classify over 10 types of drone categories with over $95 \%$ of accuracy.
\end{abstract}

\section{CCS CONCEPTS}

- Computing Methodologies $\rightarrow$ Artificial Intelligence

\section{KEYWORDS}

Convolutional Network, LeNet, drone sound, classification

\section{ACM Reference format:}

Sinwoo Yoo and Hyukjun Oh. 2020. Analysis of commercial drone sounds and its identification. In International Conference on Research in Adaptive and Convergent Systems (RACS '20), October 13-16, 2020, Gwangju, Republic of Korea. ACM, New York, NY, USA, 6 pages. https://doi.org/10.1145/3400286.3418267

\footnotetext{
Permission to make digital or hard copies of all or part of this work for personal or classroom use is granted without fee provided that copies are not made or distributed for profit or commercial advantage and that copies bear this notice and the full citationon the first page. Copyrights for components of this work owned by others than ACMmust be honored. Abstracting with credit is permitted. To copy otherwise, or republish,to post on servers or to redistribute to lists, requires prior specific permission and/or afee. Request permissions from permissions@acm.org.

RACS '20, October 13-16, 2020, Gwangju, Republic of Korea

(c) 2020 Association for Computing Machinery.

ACM ISBN 978-1-4503-8025-6/20/10..\$15.00

https://doi.org/10.1145/3400286.3418267
}

\section{INTRODUCTION}

A quadcopter drone that being propelled by 4 rotors is easy to build with reasonable prices, widely used cheap flight controllers, highly efficient BLDC motors and Lithium batteries[1] by recent market. Based on its unique and stable flight pattern it gets growing popularity in many business verticals such as filming, entertainment[2], or the new trend of the logistics market[3]. Because the lift is being generated by distributed rotors in each diagonal part the over1all size of its propeller is relatively small compares to the other similar previous types of drones such as a traditional helicopter, so this type of quadcopter drone makes very unique sounds similar to a drone we can find in nature as of its name itself. Thanks to the structural characteristics mentioned above, it is very maneuverable[4] as it sized so it is quite tricky to detect and hard to keep away from the asset we need to secure [5, 6]. Many companies in the market are investing their assets into research in order to secure a practical countermeasure with regarding to any of the possibilities of this type of flying object as being used for any of the offensive purposes or other safety issues. Some of them utilize electromagnetic radio waves to neutralize its radio link in the air as detected status, or some of them use physical methodologies to hold it down such as using nets[7, 8]. But these solutions are available or effective only if any means of preliminary awareness of approaching drones has secured. Securing practical and effective solutions for its neutralization is very hard to be flawless even in these days, and it gets worse as following the current trends of rapid improvements of the key technologies of parts themselves those consist of. One of the promising methodologies of detecting it is utilizing its unique sound pattern itself because it is very hard to eliminate the fundamental mechanism of flying and how the drone makes maneuvers. Using the sound pattern that a drone produces while it maneuvers has a limitation that it's only useful when the drone flies into the range of the sensitivity of the sensor, but still, it's very promising because it doesn't have to pinpoint the location or directions the drone approaching and also it's possible to do that with multiple sensors with certain algorithms[9]. In this manner, we performed a brief analysis with some popular commercial drones available in the market and we applied this information into the one of simplest but adjustable LeNet neural network filters with some preprocessing steps. 


\section{FUNDAMENTAL FEATURES OF THE DRONE SOUND PATTERNS AND ITS COM- PONENTS}

\subsection{Signal Characteristics}

The unique sound patterns from quadcopter drones generated by some key factors that caused by the specific structural geometry of it. One of the distinctive characteristics of a quadcopter type of drone is that does not have the direction fly the nose forward, rather be decided by only its flight controller. This is one of the main reasons that quadcopter drone can have its considerable maneuverability and also consequently the sound characteristics from most of the quadcopter drones consists of very specific several harmonics that are from each BLDC motor and propeller.

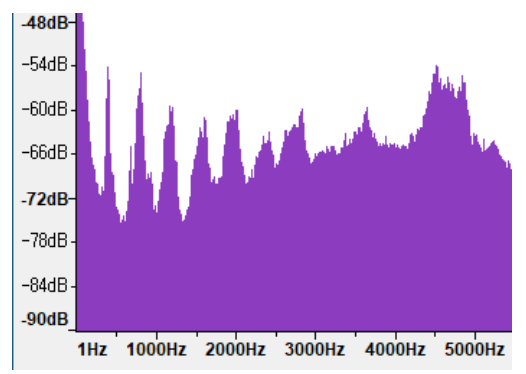

Figure 1: Frequency distribution from one of the collected sound sample with noise - Bebop 2, Parrot

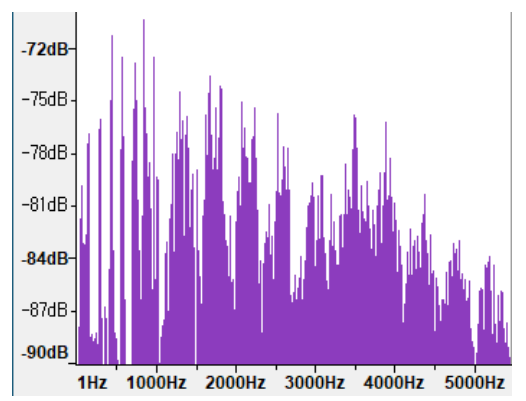

Figure 2: Frequency distribution from one of the collected sound sample - Inspire 2, DJI

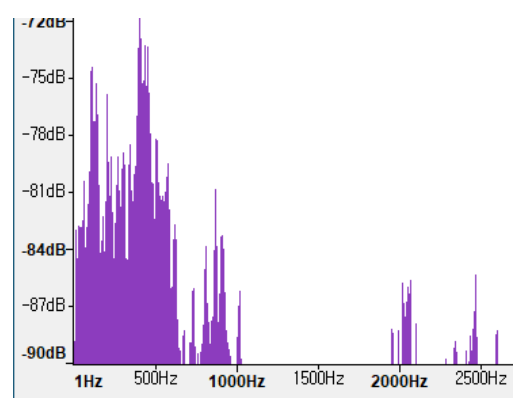

Figure 3: Frequency distribution from one of the collected sound sample - Human voice, mixed words speaking in Korean and English
Each of propellers and BLDC motors has their unique harmonics and frequency bands generated by the vibration depending on which RPM runs on during the flight controller gives a continuous signal to sustain the position with the required valance. But in given situations without dramatic changes in its altitude or hassle acceleration, it shows a very steady and certain signal power distribution which shows one of the unique characteristics that is considered for further discussion as comes up later. On the contrary, based on the biological structure of a human vocal cord, figure 3 shows clearly that human voices from words and sentences are being grouped by much gathered in narrower bands. While a quadcopter drone flying the rotating propellers hitting the air surrounding produces many other harmonics that convoluted with other adjacent frequencies besides the major ones that come directly from the moving parts themselves. Figure 2 shows a good example of the sound characteristics from most of the quadcopter drones consist of very specific several harmonics that are from each BLDC motor and propeller.

Table 1: ACOUSTIC NOISE MEASURED FOR BLDC DRIVES[10]

\begin{tabular}{rccc}
\hline $\begin{array}{c}\text { Speed } \\
(\mathrm{RPM})\end{array}$ & $\begin{array}{c}\text { Background noise } \\
\text { level }(\mathrm{dB})\end{array}$ & $\begin{array}{c}\text { Motor noise level } \\
(\mathrm{dB})\end{array}$ & $\begin{array}{c}\text { Difference } \\
(\mathrm{dB})\end{array}$ \\
\hline $\mathbf{1 0 0}$ & 48 & 72.29 & 24.29 \\
$\mathbf{3 0 0}$ & 48.3 & 75 & 26.7 \\
$\mathbf{5 0 0}$ & 49.48 & 73.54 & 24.06 \\
$\mathbf{8 0 0}$ & 47.56 & 77 & 29.44 \\
$\mathbf{1 0 0 0}$ & 50 & 82 & 32 \\
$\mathbf{1 5 0 0}$ & 48.5 & 86.52 & 38.02 \\
$\mathbf{2 0 0 0}$ & 49.23 & 89.2 & 39.97 \\
$\mathbf{2 5 0 0}$ & 52.45 & 91.26 & 38.81 \\
\hline
\end{tabular}

\subsection{Preprocessing}

The original raw wave signal that is generated from every vibrating part propagates over the air is a form of a series of samples located on the time domain, and it is hard to analyze in this form. Usually, the proper transformation process is followed as a preprocessing for the better and efficient feature distinction. A spectrogram is one effective way to obtain essential information including the power spectral density distribution among secured sampled frequencies.

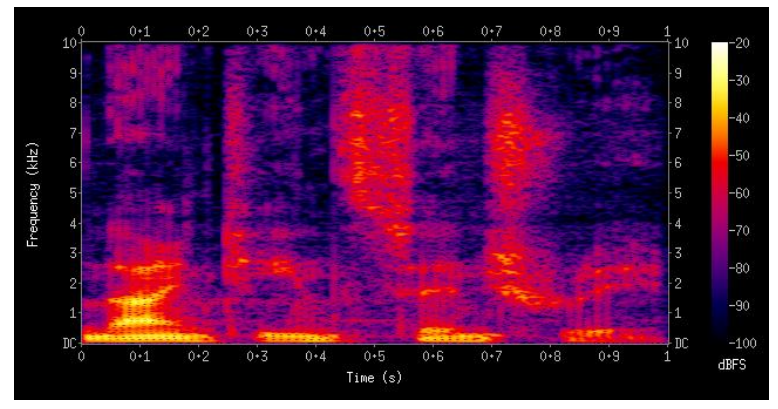

Figure 4: Spectrogram of the spoken words "nineteenth century". Frequencies are shown increasing up the vertical axis, and time on the horizontal axis, referred from Wikipedia.org 
Calculating Mel-frequency kepstrum coefficients is one of the improved methodologies from the simple spectrogram derived from the short-time Fourier transform, which has been widely utilized in especially speech recognition. Generating Melfrequency kepstrum coefficients follows certain computational steps - sample slicing, Fourier Transform, windowing, filterbank log-amplitude, Mel-scaling and smoothing, and DCT[11].
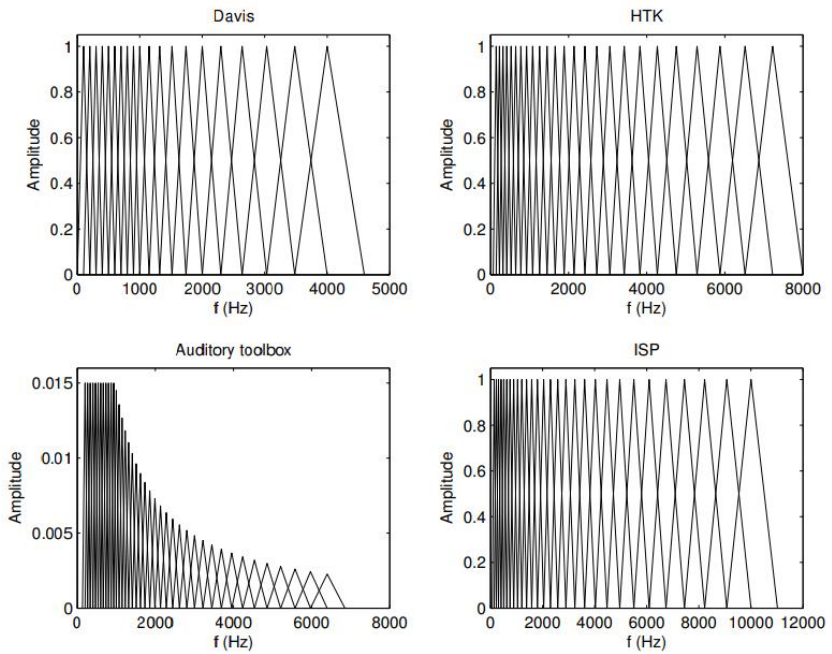

Figure 5: Shows different implementations of the Mel filter bank. Note the different scaling of the frequency axes in the plots[12]

Each of the filter-bank coefficients is obtained by suggested certain non-linear transformation including measuring windowed power spectrums from the short-time FFT into different targets or purposes of the goal. Every detail to calculate these filter-banks is available in many other papers and research[11, 12] so will not be covered in this paper. With regarding the perspective of the given sound signal as this purpose, overall frequency band from the sounds of quadcopter drones has unique harmonics but is distributed up to around $10 \mathrm{Khz}$ and their bandgaps are not wide as we observed already, so the algorithm parameters that have been used in this paper referred to HTK filter-bank but only the number of banks are selected as 40 rather than the one originally suggested 24[13] and trimmed as a fewer bin length later. While the major application that the method of preprocessing in this paper applied for is focused on specifically the human vocal sound or speech itself, the difference by the sound signals from quadcopter drones should apply needful adjustment as parameters in the preprocessing sequence.

\subsection{LeNet Neural Network}

One important foundation for applying a source signal into a certain modern neural network filter implies that there is specific information that defines the distinctive essential features within. There are many suggested and practically proven neural network models already in speech recognition and already widely being used for many services effectively yet but due to the significant differences mentioned above, there are no practical applications for the sound signal for the ones of quadcopter type of drones. There have been in-depth studies previously for the past several years, but unfortunately, I carefully consider that some of them are hard to be reorganized or being shaped in an efficient and robust performance yet[14-16].

LeNet neural network model was announced in 1989 with its first original structure and shows its performance with the meaningful efficient network model as introducing a convolutional neural network for the first time[17]. Multiple twodimensional kernel planes as converging feature extractors operate as the key component by a unique concatenated convolutional computation sequence. One of its promising foundations that makes LeNet neural network filter effective is that this architecture maintains the spatial information by given the 2nd order as Euclidian distances during the learning process, and the information that is included among the raw input data does deliver the weights of the kernels gradually during the following optimization process and backpropagation updates effectively. LeNet has several hyperparameters that could be adjusted for better outcomes and training results depending on the given typical sets of possible datasets. It is very hard to estimate how much of the capacity the neural network filter has for the given learning optimization process, and it doesn't exist yet any of practical metrics to measure the upper bound of the learning capacity of any given neural network which can be indicated as a structured complexity level of nonlinearity. Therefore, using LeNet in this analysis should be assumed that has no issues upon the perspective of its limit including possible stochastic deductions of measurable elements throughout the whole learning process[18]. Considering used computation elements among this network as for the kernel sizes and lengths of the fully connected layers, we can try to estimate how much of capacity could be considered from the given network. The range of kernel filter size was from 5 up to 10 square pixels, the size which notated as $x$ and $y$, each of kernel filter pixel can be obtained by the iterations from the bidirectional propagations, activation function $f_{\text {act }}$ is essential to make possible stacking nonlinearity through the propagations of entire synapse elements (1).

$$
\begin{gathered}
K_{x, y}^{i}=f_{a c t}\left(\left(W^{i} * x\right)_{x, y}+b_{i}\right) \\
\mathrm{K}_{\mathrm{m} \times \mathrm{n}}^{\mathrm{i}}[\mathrm{x}, \mathrm{y}]=\operatorname{img}[\mathrm{x}, \mathrm{y}] *\left[W_{r^{i} \cdot x, r^{i} \cdot y}^{i}\right]
\end{gathered}
$$

The input image size $\operatorname{img}[x, y]$ denotes its width and height as $x$ and $y$, and it defines the size of the calculated kernel filter. The ratio $r^{i}$ denotes how much of kernel filter elements were being operated for the forward propagation operations to the right answer at the $i^{\text {th }}$ kernel filter, which is applied to the given kernel filter size. It can also be referred to as the kernel size of the extracted $m \times n$ feature map (2). The proper learning process can be achieved only if the information in the given input signal is smaller than the size of this kernel, and the overlapping window length that used for the MFCC preprocessing as mentioned before is being expected to be longer than the tested range of the kernel sizes. Each of sample pixel indicates the length of the FFT window, it ranges to have up to $40 \mathrm{~ms}$ from $10 \mathrm{~ms}$ at least through 
the entire tests. In one of test case that has the hyperparameter values such as 8 square pixels of 1st convolutional stage of 32 kernel filters, 10 square pixels of $2^{\text {nd }}$ convolutional stage of 64 kernel filters, 1024 elements of $3^{\text {rd }}$ stage of fully connected dense layer stage, and $4^{\text {th }}$ of 10 classes of final logit outputs would have the number of the total possible computation elements can be calculated as $\cong 1.34 \mathrm{M}$ elements. Its total possible states can be obtained by multiplying the each of bit-widths of variables that are used for the network implementation, it could be 8 bits of the grayscale pixel or 32 bits of floating-point variables in range. This is just a simple estimation only considering with total computational state numbers and neuron elements which directly affect the initial positive learning chances and effective accumulations for total output value from the cost function.

\section{EXPERIMENTAL AND COMPUTATIONAL DETAILS}

\subsection{Preprocessing}

The unique sound patterns from quadcopter drones generate a key feature of it by the specific geometrical structure as its flight and maneuvering. While a human vocal cord is not a static or hardly casted material so it could distort the majority tones with slight frequency dispersions or spreads respectively, a quadcopter drone has very solid and fixed geometry and distinctive signal sources such as BLDC motors, propellers, arms, and its sculpture as regarding most of the drones that are available in the commercial market. Still, all variations of RPM controlling, and intended maneuvers produce respective frequency slides and dopplers consequently, but it is considerably limited comparing any other sources from nature. One of the distinctive differences we could peek from the overall spectrum analysis in order to find the proper way of utilizing Mel-frequency kepstrum coefficients algorithm as the preprocessing for a dimension reduction with the input signal is that the distribution of the power spectral density shows much more arranged and stable harmonics comparing to the human vocal sound, and these narrow bands of each group of tones allow us to reduce filter-bank gaps down while expanding the upper side of the cutoff frequency. Each of the frequency bins was prepared as 40 triangular shaped band filters and calculating the log-energy amplitudes and frequencies for each bandgap are followed by equations as below.

$$
\begin{gathered}
m=2595 \times \log _{10}[1+f / 700] \\
\mathrm{f}=700 \times\left(10^{\mathrm{m} / 2595}-1\right)
\end{gathered}
$$

There will 13 overlaps for the frequency bands up to $1 \mathrm{KHz}$ from $133.3 \mathrm{~Hz}$ for the $50 \%$ of linear dividing, while 27 for the band gaps from 1 to $8 \mathrm{Khz}$ for logarithmical filter spaces. It varies depending on how much of cut-off frequency has been set[13].

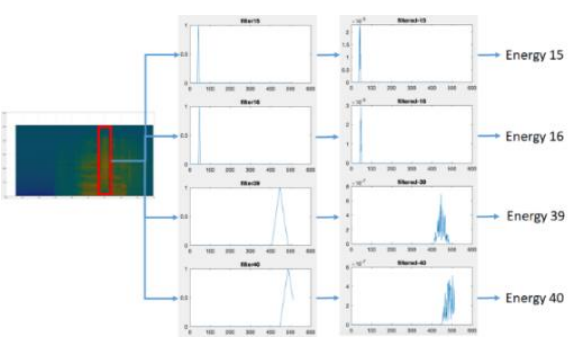

Figure 6: Tried to apply different cases of filter-banks and Mel-frequency bin selection. We tried them up to 40 and extracted bins fewer than 10

Since of many practical limitations to build the evenly distributed dataset as regarding the type of the source signal we need to consider that this empirical selection could be biased or not well arranged, but the simulator used in this paper was designed with the utmost flexibility that allowed and able to adjust its hyperparameters as below, thanks to the convolutional neural network model that has the essential architecture of keeping the information that is distributed on time and spatial domain in both. In order to provide the maximal concentration among the source signal features at least there some picked wave clips were selected and trimmed from the raw wave signals, but the majority portion of the raw input wave sources includes strong white and biased ambient noises in overall.

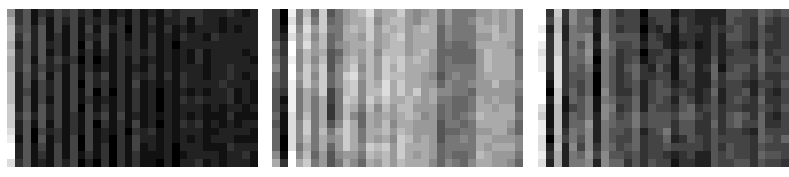

Figure 7: MFCC spectrograms that were used for input vectors. Selected 32 bins from generated total 40 frequency bins, for 20 samples by 1024 SFFT points under $48 \mathrm{Khz}$ sample rates. From the left, Background noise, Bebop 2 (Parrot), and F450 (DJI, Custom)

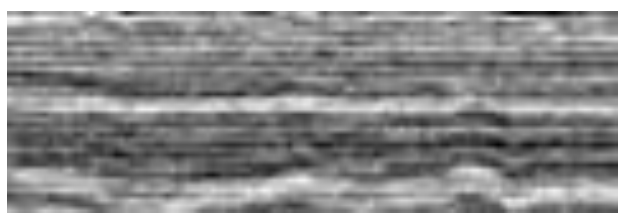

Figure 8: MFCC spectrogram, Mavic Air from DJI, Hovering

Table 2: All tested preprocessing parameters

\begin{tabular}{ll}
\hline Test Parameters & Configurations \\
\hline Sample rate & $44.1 \sim 48 \mathrm{Khz}$ \\
Normalization & Ortho-normal DCT \\
Mel-Frequency bins & $8 \sim 40$ \\
Stride window & FFT size / 2 \\
STFT length & $512 \sim 2048$ \\
MFCC frame window & $10.6 \sim 42.6 \mathrm{~ms}$ \\
Spectrogram dimension & $(8 \sim 40) \times(512 \sim 2048)$ \\
\hline
\end{tabular}


Librosa which is one of the popular open-sourced projects for music and audio signal analysis was utilized for the complete computation sequences to obtain Mel-frequency Kepstrum coefficients from the given raw wave signals. Based on the overall signal pattern that was used for the input ground fact as recorded as the noisy and rough environment in this analysis and experiment, using optimizers that don't have relative momentum factors inside didn't help for effective training while the other ones have. Due to the completely limited and reduced the total number of the calculation elements consists of the entire this neural network which was intended as a means to apply the following evaluation on NVIDIA Jetson Xavier as the target device, we downsized the resolution of the input signal as under 1000 pixels and now concatenated short-term FFT coefficients keep being influenced by the distributed power signal of other adjacent frequency bands, while and non-white noises. Pixels within the size of the kernels for the convolutional computation but doesn't have any adjacent pixels in a time-domain within the equivalent period of the FFT size could be considered as noise elements from low SNRs or some erupted noise such like winds, human voices, car engines noise, bird chirps, and etcetera. Since most of the waveforms that used as input vectors for the network have their unique buzzing sound pattern long enough, as long as we can recognize any of straight or curved "lines" from Mel-frequency kepstrum coefficient spectrograms in a shape of 2-d planes input vectors, we can expect that this is what the convolutional kernels may find their own way of shaping along with the results from the cost function and following optimizer algorithm.

\subsection{Training}

Entire training and evaluation were designed and performed by using Google TensorFlow framework and other python libraries, and overall trends on the training timeline are shown as figures 9 and 10, respectively. Including one eraser category, prepared sample classes are 10 types of recorded drone sounds, over 2.4 Gbytes of stereo wave files were used for this supervised learning. Many different variances by combinations with preprocessed input signals were applied, and the overall learning process is assessed by updated weights through the summation of cross-entropy losses, and the following accuracy showed quite promising results.

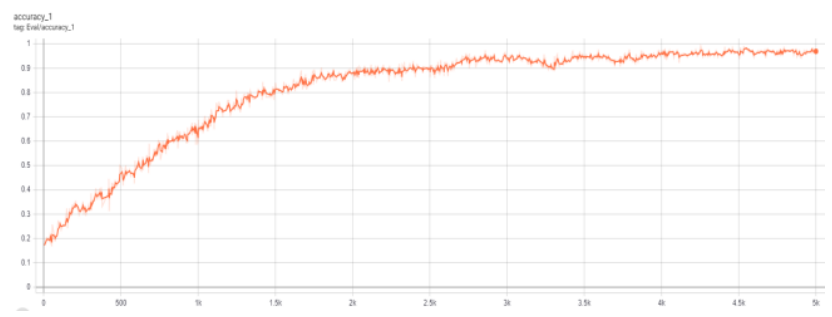

Figure 9: Accuracy trend timeline with ADAM optimizer, learning rate
Table 3: All tested parameters for the performed training

\begin{tabular}{ll}
\hline Test Parameters & Configurations \\
\hline Optimizers & Adam, adadelta, adagradDA \\
Batch sizes & $128 \sim 256$ \\
Cost function & Cross entropy sigma with 11 logits \\
framework & Tensorflow \\
\hline
\end{tabular}

The learning process was performed with several types of optimizer algorithms that prepared on Google TensorFlow library AdamOptimizer, AdadeltaOptimizer, and AdagradDAOptimizer. The size of the batch regularization window was also applied in some different combinations from 128 to 256 max. Only softmax cross-entropy with multiple outputs was used to calculate the endpoint costs with the one-hot encoding labels.

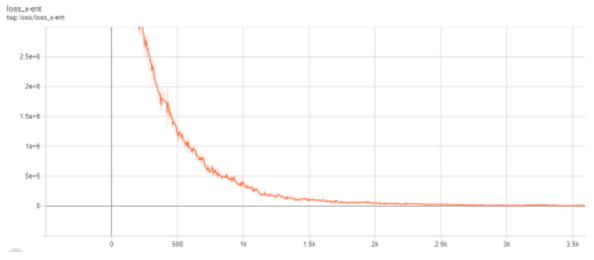

Figure 10: Trend of decreasing calculated loss from the cost function - merged 10 logits and 1 eraser by crossentropy function

By analyzing recorded graphs and scalar vectors as with weights and biases from training progresses taking an example of it as Figure 11, we could assume that this network does not use all its total computational elements yet. The overall distribution we could see there are clearly zero-valued element counts throughout the network elements even after its saturation, which means that the applied dropout sequence neutralizes considerable number of synapses to secure a certain level of generalizations.

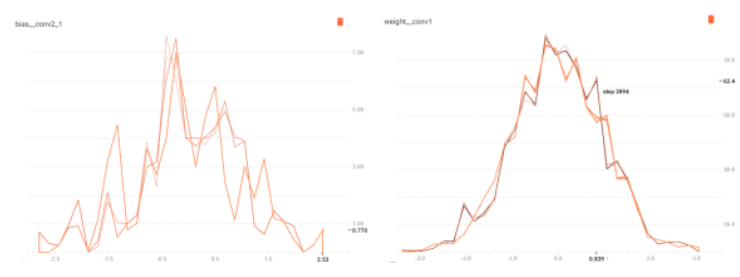

Figure 11: Tensorboard records show there are zero-valued elements were recorded and its ratio are zero centered. Bias values on the left is a comparable reference as a linear factor.

But one thing that we need to be sure of is that the convolutional computation sequence does not transform any of the information from the input stream continuously provided into another degree or dimension of a distinct set of the group, such as a linguistic word or vector set. Unlike some other network that has the outputs as totally altered or reduced dimensions from the input signals, such as autoencoder models or many variances of recursive networks, only extracted features from the input exist on the 2-d plan kernels which have fixed coordinates as the time 
and frequency domain. Hence, if we expect the same result of the training progress, it is only able to be effective as long as the signal sources can be placed on the absolutely same Euclidean plane, which means if we need to use these outcomes into a practical application by signal evaluation, that signal should be obtained by the same quadcopter drones but also by exactly the same sensors. With the existence of any possible non-linear factors within the overall signal flow through the given preprocessing or the prepared neural network, we cannot rely on the extracted features anymore. A signal processing sequence itself is not able to be implemented without nonlinear factors or any distortion from it and this case is also inevitable. This is the reason that the general speech recognition model generates the corresponding words or some of the vector subsets, while the voice signal classification does extract voice biometrics which can be regarded as a memorizing process.

\section{RESULT AND DISCUSSION}

\subsection{Overall performance}

After around $5 \mathrm{~K}$ iteration as repeated with 128 to 256 batch-sized regularizations and up to $40 \%$ of dropout ratio from $20 \%$ the model shows a decent accuracy, around $96 \%$ overall. Some of the learning cases show some overfitted situation around $20 \%$ of the dropout ratio, but at the point of around $40 \%$ of dropout achieved the maximum accuracy in a stable with repeated training and testing. Figure 9 shows the overall learning curves during the iteration, and figure 10 is for the sum of cross-entropy error from the 11 logits output in the same way as the previous one.

\subsection{Consideration}

As expected, weights and biases on kernels of the convolutional filters can be adapted by specific features for the input signal patterns. With regarding spatial information, dealing with these sound signals into 2-d planning is obviously reasonable and has been justified. Therefore, we can consider that the overall outcomes and its performance seem quite promising to utilize it towards other networks or applications.

Further research will cover the better hybrid neural network model over the larger computational elements within in order to overcome the input signal sensor sensitivity including without the alternation of information dimensions or preprocess sequences.

\section{ACKNOWLEDGMENTS}

The authors gratefully acknowledge the support from Nano UAV Intelligence Systems Research Laboratory at Kwangwoon University, originally funded by Korean Defense Acquisition Program Administration (DAPA) and Agency for Defense Development (ADD).

\section{REFERENCES}

[1] DW Lee. 2014. Development of BLDC motor and multi-blade fan for HEV battery cooling system. International fournal of Automotive Technology 15, 7 (2014), 1101-1106.

[2] Ioannis Mademlis, Vasileios Mygdalis, Chara Raptopoulou, Nikos Nikolaidis, Nicolaus Heise, Tim Koch, Jan Grunfeld, Tilman Wagner, Alberto Messina,
Fulvio Negro, et al. 2017. Overview of drone cinematography for sports filming. In European Conference on Visual Media Production (CVMP), short.

[3] Chase C Murray and Amanda G Chu. 2015. The flying sidekick traveling salesman problem: Optimization of drone-assisted parcel delivery. Transportation Research Part C: Emerging Technologies 54 (2015), 86-109.

[4] Azade Fotouhi, Ming Ding, and Mahbub Hassan. 2017. Understanding autonomous drone maneuverability for internet of things applications. In 2017 IEEE 18th International Symposium on A World of Wireless, Mobile and Multimedia Networks(WoWMoM). IEEE, 1-6.

[5] Tomasz Sosnowski, Grzegorz Bieszczad, Henryk Madura, and Mariusz Kastek. 2018.Thermovision system for flying objects detection. In 2018 Baltic URSI Symposium(URSI). IEEE, 141-144.

[6] Martins Ezuma, Ozgur Ozdemir, Chethan Kumar Anjinappa, Wahab Ali Gulzar, and Ismail Guvenc. 2019. Micro-UAV detection with a low-grazing angle millimeter wave radar. In 2019 IEEE Radio and Wireless Symposium (RWS) IEEE, 1-4.

[7] Veli-Pekka Kutinlahti et al. 2019. Antenna for Directive Energy Device Against Drones. (2019).

[8] Gabriel C Birch, John C Griffin, and Matthew K Erdman. 2015. UAS Detection, Classification, and Neutralization: Market Survey 2015. Sandia National Laboratories (2015).

[9] Thyagaraju Damarla. 2015. Detection of Gunshots using Microphone Array mounted on a moving Platform. In 2015 IEEE SENSORS. IEEE, 1-4.

[10] RM Pindoriya, AK Mishra, BS Rajpurohit, and R Kumar. 2018. An analysis of vibration and acoustic noise of BLDC motor drive. In 2018 IEEE Power \& Energy Society General Meeting (PESGM). IEEE, 1-5.

[11] Beth Logan et al. 2000. Mel frequency cepstral coefficients for music modeling.. In Ismir, Vol. 270. 1-11.

[12] Sigurdur Sigurdsson, Kaare Brandt Petersen, and Tue Lehn-Schiøler. 2006. Mel Frequency Cepstral Coefficients: An Evaluation of Robustness of MP3 Encoded Music.. In ISMIR. 286-289.

[13] Todor Ganchev, Nikos Fakotakis, and George Kokkinakis. 2005. Comparative evaluation of various MFCC implementations on the speaker verification task. In Proceedings of the SPECOM, Vol. 1. 191-194.

[14] Juhyun Kim and Dongho Kim. 2018. Neural network based real-time UAV detection and analysis by sound. Fournal of Advanced Information Technology and Convergence 8, 1 (2018), 43-52.

[15] Sungho Jeon, Jong-Woo Shin, Young-Jun Lee, Woong-Hee Kim, YoungHyoun Kwon, and Hae-Yong Yang. 2017. Empirical study of drone sound detection in real-life environment with deep neural networks. In 2017 25th European Signal Processing Conference (EUSIPCO). IEEE, 1858-1862.

[16] Adrian Carrio, Carlos Sampedro, Alejandro Rodriguez-Ramos, and Pascual Campoy. 2017. A review of deep learning methods and applications for unmanned aerial vehicles. fournal of Sensors 2017 (2017).

[17] Yoshua Bengio, Yann LeCun, et al. 2007. Scaling learning algorithms towards AI. Large-scale kernel machines 34, 5 (2007), 1-41.

[18] Alessandro Achille and Stefano Soatto. 2018. Information dropout: Learning optimal representations through noisy computation. IEEE transactions on pattern analysis and machine intelligence 40, 12 (2018), 2897-2905. 